\title{
Oral health in prevalent types of Ehlers-Danlos syndromes
}

\author{
Peter J. De Coster ${ }^{1}$, Luc C. Martens ${ }^{1}$, Anne De Paepe ${ }^{2}$ \\ ${ }^{1}$ Department of Paediatric Dentistry, Centre for Special Care, Paecamed Research, Ghent University, Ghent; ${ }^{2}$ Centre for Medical \\ Genetics, Ghent University Hospital, Ghent, Belgium
}

\begin{abstract}
BACKGROUND: The Ehlers-Danlos syndromes (EDS) comprise a heterogenous group of heritable disorders of connective tissue, characterized by joint hypermobility, skin hyperextensibility and tissue fragility. Most EDS types are caused by mutations in genes encoding different types of collagen or enzymes, essential for normal processing of collagen.

METHODS: Oral health was assessed in 31 subjects with EDS ( 16 with hypermobility EDS, nine with classical EDS and six with vascular EDS), including signs and symptoms of temporomandibular disorders (TMD), alterations of dental hard tissues, oral mucosa and periodontium, and was compared with matched controls.

RESULTS: All EDS subjects were symptomatic for TMD and reported recurrent temporomandibular joint (TMJ) dislocations. Abnormal pulp shape (13\%) and pulp calcification (78\%) were observed in subjects affected with classical EDS. Caries experience was higher in EDS compared with controls and was related to poor oral hygiene, influenced by increased mucosal fragility and restraint of (wrist) joint mobility. The overall periodontal status in EDS was poor, with $62 \%$ of EDS subjects presenting high periodontal treatment needs (community periodontal index for treatment need, CPITN = II).

CONCLUSION: Oral health may be severely compromised in EDS as a result of specific alterations of collagen in orofacial structures. When considering dental treatment in EDS, a number of tissue responses (mucosa, periodontium, pulp) and precautions (TMJ dislocation) should be anticipated.

J Oral Pathol Med (2005) 34: 298-307
\end{abstract}

Keywords: collagen metabolism; Ehlers-Danlos syndrome; oral health; periodontal connective tissues; temporomandibular disorders

Correspondence: Dr Peter De Coster, Department of Paediatric Dentistry, Centre for Special Care, Ghent University Hospital - 0P8, De Pintelaan 185, B-9000 Ghent, Belgium. Tel: + 32-9-240-40-08, Fax: +32-9-240-38-51, E-mail: luc.martens@ugent.be

Accepted for publication October 11, 2004

\section{Introduction}

The Ehlers-Danlos syndromes (EDS) comprise a heterogenous group of heritable disorders of connective tissue, largely characterized by joint hypermobility, skin hyperextensibility and tissue fragility (1) (Fig. 1). The clinical features, modes of inheritance and molecular bases differ according to the type. EDS are caused by a genetic defect causing an error in the synthesis or processing of collagen types I, III or V. The distribution and function of these collagen types are displayed in Table 1. At present, two classifications of EDS are commonly used of which the most recent taxonomy is largely based on recent developments in the elucidation of the biochemical and molecular bases of the disorder (2). As displayed in Table 2, more than 10 subconditions were delineated according to differences in clinical features, modes of inheritance and biochemical defects. According to the most recent classification (2), six clinical features are commonly shared with varying expression and varying diagnostic weight by the different types: skin hyperextensibility, generalized joint hypermobility, easy bruising, tissue fragility, mitral valve prolapse, proximal aorta dilatation, and chronic joint and limb pain. The most recent classification discriminates the classical type (OMIM Entry 130000 130010) (3), hypermobility type (OMIM Entry 130020), vascular type (OMIM Entry 130050), kyphoscoliosis type (OMIM Entry 225400), arthrochalasia type (OMIM Entry 130060) and dermatosparaxis type (OMIM Entry 225410) as chief conditions of EDS. In terms of prevalence, hypermobility EDS type is thought to occur in $80 \%$ of EDS, classical type in $10 \%$, whereas the other types are assigned a lower frequency. Although there is conflicting evidence in literature concerning the prevalence of the distinct EDS types, estimates of approximately 1:10 000-20 000 for classical EDS type (former EDS types I and II), and 1:100 000 for vascular EDS type (former EDS type IV) have been reported in various studies (4). The actual incidence, however, may be fairly greater as diagnostic skills at the molecular/biochemical level are steadily improving.

Oral abnormalities were first reported by Barabas and Barabas (5), and may include small, irregularly placed teeth in the presence of partial anodontia $(6,7)$ or 

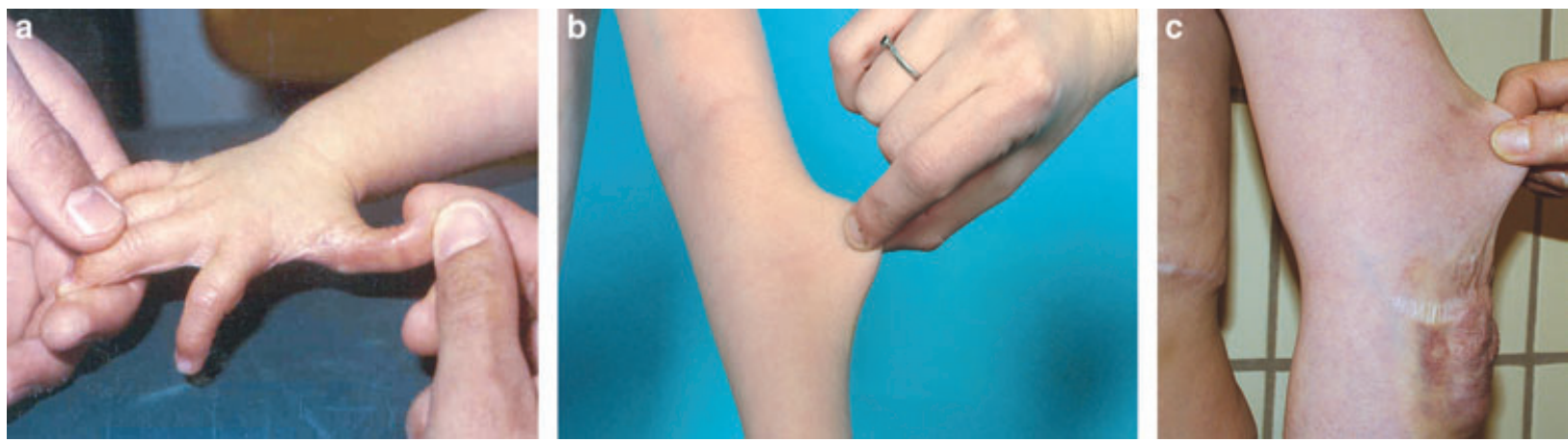

Figure 1 Hypermobility of the joints (a), skin hyperextensibility (b) and increased tissue fragility with extensive scarring (c) are pathognomic features of Ehlers-Danlos syndromes (EDS).

Table 1 Distribution and function of the major fibrillar collagens

\begin{tabular}{|c|c|c|}
\hline Collagen type & Function & Distribution \\
\hline Collagen I & $\begin{array}{l}\text { Providing functional tissues with a structural framework } \\
\text { Initialization of mineralization in calcified tissues }\end{array}$ & Bone, muscle, ligaments, skin, mucosae, organs \\
\hline Collagen III & $\begin{array}{l}\text { Scar formation } \\
\text { Transition to late mineralization stage in calcified tissues } \\
\text { Providing elastic properties to vessel walls }\end{array}$ & Bone, ligaments, skin, organs, vessels \\
\hline Collagen V & Regulation of fibril formation of collagen I & Bone, muscle, ligaments, skin \\
\hline
\end{tabular}

Table 2 Current classifications of Ehlers-Danlos syndromes (EDS)

\begin{tabular}{|c|c|c|c|}
\hline New 'Villefranche' classification ${ }^{\mathrm{a}}$ & Former 'Berlin' classification ${ }^{\mathrm{b}}$ & $O M I M^{\mathrm{c}}$ & Inheritance ${ }^{\mathrm{d}}$ \\
\hline \multirow[t]{2}{*}{ Classical type } & Gravis (EDS type I) & 130000 & $\mathrm{AD}$ \\
\hline & Mitis (EDS type II) & 130010 & AD \\
\hline Hypermobility type & Hypermobile (EDS type III) & 130020 & $\mathrm{AD}$ \\
\hline Vascular type & Arterial-ecchymotic (EDS type IV) & 130050 & $\mathrm{AD}$ \\
\hline Kyphoscoliosis type & Ocular-scoliotic (EDS type VI) & 225400 & $\mathrm{AR}$ \\
\hline Arthrochalasia type & Arthrochalasis multiplex (EDS types VIIA and VIIB) & 130060 & $\mathrm{AD}$ \\
\hline Dermatosparaxis type & Human dermatosparaxis (EDS type VIIC) & 225410 & AR \\
\hline \multirow{5}{*}{ Other forms } & X-linked EDS (EDS type V) & 305200 & $\mathrm{XL}$ \\
\hline & Periodontitis type (EDS type VIII) & 130080 & $\mathrm{AD}$ \\
\hline & Fibronectin-deficient type (EDS type X) & 225310 & ? \\
\hline & Familial hypermobility syndrome (EDS type XI) & 147900 & $\mathrm{AD}$ \\
\hline & Progeroid EDS & 130070 & $?$ \\
\hline
\end{tabular}

a'Villefranche' classification (2).

b'Berlin' classification (1).

'OMIM Entry (Online Mendelian Inheritance in Man). Available at: http://www3.ncbi.nlm.nih.gov/OMIM (3).

${ }^{\mathrm{d}} \mathrm{AD}$, autosomal dominant; AR, autosomal recessive; XL, sex linked.

multiple supernumerary teeth (8). Temporomandibular joint (TMJ) dysfunction has also been described in some patients (9-15). Severe periodontal disease was reported to be a pathognomic feature of vascular EDS type (former type VIII) $(16,17)$, and defective dentinogenesis, pulp and root deformities have been reported in classical EDS type (former type I) (18). Apical root resorption after orthodontic treatment has been reported in vascular EDS (19). Most studies, however, have documented on a small number of cases and failed to establish the EDS type by means of a biochemical/molecular diagnosis. As a consequence, the diagnostic weight of these oral findings can be questioned.

The aim of this study was to analyse the different manifestations in the orofacial region and to assess oral health in a population consisting of the three most prevalent EDS types (classical, hypermobility and vascular types). In addition, a series of guidelines is proposed to integrated in dental treatment strategies of patients affected with EDS.

\section{Material and methods}

\section{Material}

The study group comprised 31 patients $(n=31)$ who were diagnosed according to the above-mentioned criteria (2) at the Centre for Medical Genetics, Ghent University Hospital, Belgium. The EDS subtype distribution was as follows: 16 subjects had hypermobile EDS (former EDS type III), nine had classical EDS (former 
types I and II) and six had vascular EDS (former type IV). Mean age of the study group was $28 \pm 15.3$ years (age range: 4-61), and gender distribution was 35\% males to $65 \%$ females. Forty-nine healthy subjects $(n=49)$, presenting for general dentistry purposes and without a history of cardiovascular, endocrine, haematological or infectious diseases, were enrolled in a control group. The control subjects were matched for age, sex and dental attendance. Mean age of the control population was $29.2 \pm 15.9$ years (age range: 5-62), and sex distribution was $39 \%$ males to $61 \%$ females.

\section{Methods}

All subjects were examined and interviewed by the same trained investigator (PDC), using standardized criteria as recommended by the WHO's report on oral health surveys (17). The oral assessment included the following sections: general medical information, extra-oral information, TMJ assessment, oral mucosa, enamel opacities/hypoplasia, caries experience and oral cleanliness, periodontal status, loss of gingival attachment, frequency of visiting the dental office and prosthetic status (20). In view of analysing previously reported phenomena in the syndrome, the WHO oral assessment was extended with structured queries on TMJ dislocation and the condition of the oral mucosa (including occurrence, initiating factors and management of increased mucosal fragility). Panoramic radiographs and bitewings (taken with standardized Rinn-device) were examined for anomalies of tooth number, shape and structures.

Assessment of general medical status included registration of EDS type and acute and chronically administered medication. Extra-oral information comprised clinical assessment of extra-oral ulcerations, swellings, sores, erosions, scars or fissures. Signs and symptoms of temporomandibular disorders (TMD) were recorded during clinical examination and patient interview, based on the Research Diagnostic Criteria (RDC) for TMD $(21,22)$. Subjects with muscle spasm, myositis, contracture, or a history of whiplash, facial trauma (presence of facial scars), rheumatic disease and/or arthritis of the neck-shoulder complex were excluded for examination, as these conditions may mimic orofacial pain. Four indicators were evaluated for TMD diagnosis: orofacial pain, joint sounds, range of mandibular opening and radiographic evaluation of joint surfaces. Orofacial pain was recorded as originating either from the masticatory muscles or from the joint capsula, including a report of pain in the jaw, temples, face, pre-auricular area or the TMJ both at rest or in function, or tenderness in response to palpation of the masticatory muscles (muscle pain) and/or the TMJ (joint pain). The range of mandibular opening was measured by use of callipers both in vertical (opening) and horizontal direction (lateral), taken as the average distance between the incisal tips of the central maxillary and mandibular incisors on three consecutive trials. The distances were registered during pain-free unassisted (active range of movement) and assisted opening movement (passive range of movement). Joint sounds of one or both TMJs were recorded when a sharp sound (clicking or crepitus) was present (audible or by palpation of the lateral TMJ pole) during both opening and lateral movements on at least three consecutive trials. The joint surfaces were evaluated on panoramic radiographs for extensive flattening of the surfaces, osteophyte formation or sclerosis of the joint parts. The clinical indicators allowed for the possibility of multiple TMD diagnoses for a given subject. TMD diagnoses were divided into three groups: muscle disorders (group I), disc disorders (group II) and joint disorders (group III) (21). Oral parafunctions (grinding teeth, clenching, crushing Popsicles, etc.) and psychosocial factors (family history, emotional or social problems, susceptibility to stress) were analysed as candidate factors predisposing to development and perpetuation of TMD $(21,22)$. Characteristics of TMJ dislocation were recorded during patient interview, comprising frequency of dislocation events, provoking factors, and duration, reduction, and consequences of dislocations. A TMJ dislocation, also known as open lock or subluxation, was defined as a condition in which the condyle is positioned anterior to the articular eminence, and is unable to return to a closed position. It is manifested clinically as an inability to close the mouth without a specific manipulative manoeuvre. Dislocation may be the result of a physical jamming of the disc-condyle complex beyond the articular eminence, i.e. maintained by muscle activity or a true hyperextension of the disc-condyle complex beyond its normal translation position (23).

The condition of the oral mucosa was assessed by recording evidence of increased mucosal fragility (bruises, grazes, regenerative patches or ulcerations) at different locations of the lining (floor of the mouth, underside of the tongue, inside of the lips, soft palate, cheeks and alveolar processes) and masticatory mucosa (hard palate and gingiva). Bruises were defined as dark coloured (red to blueish-grey) mucosal swellings, not related to dental/periodontal pathology or neoplasmata, scar tissue or hyperpigmentation. Grazes were defined as circumscribed, dark red or purple coloured areas, usually displaying exposure of capillary vessels, indicated by the presence of small red points. Regenerative patches were defined as circumscribed spots, either of white, grey or brown colour, usually with a paler outline and reflecting recent bruising and/or erosion with deficient wound healing. These patches should be distinguished from abscesses and ulcerations (aphthous, herpetic or traumatic), and mucosal conditions with a more durable character such as leukoplakia, lichen planus and candidiasis. A positive history of recurrent mucosal trauma was required to confirm the clinical diagnosis of increased mucosal fragility. In these cases, the occurrence (daily; once or more a week; less), initiating factors (mastication; brushing teeth; consumption of hot, spicy or crusty foods or candy; spontaneously), and individual management (nihil; mouth rinses; topical gels; professional help) of the lesions were scored on an ordinal scale.

Enamel abnormalities were classified into three types on the basis of their appearance: demarcated opacities, 
diffuse opacities and hypolasia (20). Caries experience was assessed using the dmf-s/DMF-s index, recording decay at the level of cavitation. All teeth except third molars were scored using a $\mathrm{WHO} /$ community periodontal index for treatment need (CPITN) type E probe, a white light source, cotton rolls and a dental mirror (24). The dental care index (Jackson's Restorative Index) was expressed as $F / D+F(25)$. Visual presence of plaque was scored on tooth surface level using Silness \& Loë's Plaque Index system (PII) (26). The periodontal status was assessed by use of two indicators: Loë \& Silness' Gingival Index system (GI) (26) and probing depth of periodontal pockets. A blunt WHO/CPITN type E probe with an end diameter of $0.5 \mathrm{~mm}$ was used for examination of the tooth surfaces and gingival attachment $(20,24,26)$. Information on loss of gingival attachment was collected by probing pocket depths of all teeth except third molars, permitting comparison between population groups, without the intention, however, to describe the individual extent of loss of attachment (20). Probing pocket depth was not recorded for children under the age of 14 years. Periodontal treatment needs were indicated on a scale of 0 -III on the basis of the CPITN score $(26,27)$. Oral care and oral hygiene habits were recorded on an ordinal scale by use of three indicators: dental attendance, brushing frequency, and the impact of both the physical and the mucosal condition on brushing habits.

\section{Examinator reliability}

The examiner was calibrated at baseline for scoring DMF-s, plaque (Silness \& Loë's PII system), gingival inflammation (Loë \& Silness' GI system), CPITN, and symptoms and signs of TMDs (RDC for TMD). Intraand inter-examiner consistency was tested for these scoring methods by comparing a number of paired measurements.

\section{Statistics}

Statistics were performed using Fischer's exact test and chi-square test for comparison of proportions, MannWhitney $U$-test (unpaired Wilcoxon test) to compare mean values between two groups, one-way ANOVA to analyse the effect of qualitative factors on continuous variables, and rank correlation analysis (Spearman's correlation coefficient) and regression analysis to describe the relationship between two continuous variables. Differences at the $P \leq 0.05$ level were considered statistically significant $(28,29)$.

The study design had previously been approved by the Ethical Committee of Ghent University Hospital (ref. 2000/309). A written consent was obtained from all participants prior to examination.

\section{Results}

\section{Reliability}

Intra-examiner agreement was tested using Kappastatistics (Cohen's Kappa or $\kappa$ ) for DMF-s score $(\kappa=0.78)$. Inter-examiner agreement was tested for probing pocket depth $(\kappa=0.96)$, and scoring gingival inflammation (Silness \& Loë's GI) $(\kappa=0.83)$, dental plaque (Silness \& Loë's PII) $(\kappa=0.88)$, palpation for muscle (at 16 different sites; $\kappa=0.60$ ) and joint tenderness (lateral and posterior pole; $\kappa=0.78$ ), evaluation of the quality and occurrence of joint sounds on vertical opening $(\kappa=0.69)$ and with excursive movements $(\kappa=0.65)$, and registration of the jaw opening pattern $(\kappa=0.78)$. These Kappa-values stand for an acceptable inter-reliability with an experienced examiner as a benchmark. Intraclass correlation coefficients (ICC) were computed for continuous variables $(28,29)$, such as linear measurements of mandibular border positions, and ranged from 0.92 to 0.99 , indicating excellent reliability between two examiners.

\section{Medical status}

Limb and/or joint pain was reported in $67.7 \%$ of EDS population, manifesting in an acute or chronic (circadian) pattern. Chronic TMJ pain was a frequent finding in over $50 \%$ of EDS. All subjects with limb or joint pain were $\geq 18$ years of age, and no gender difference was found with regard to pain report $(P=1.0)$. In $48.4 \%$ of EDS population, chronic pain was recorded in the presence of mild-to-severe restraint of physical activities. There was no difference in reporting of restraint mobility between males and females $(P=0.456)$. No differences were found between EDS types in frequency of reports of pain $(P=0.285)$ or restraint joint mobility $(P=0.441)$. Chronical administration of non-steroidal anti-inflammatory drugs was recorded in $29 \%$ of EDS population to relief chronic limb and joint pain.

\section{Temporomandibular disorders}

About $83 \%$ of the subjects were assigned myofascial pain diagnosis (group I), whereas unilateral and bilateral disc displacement with reduction (group II) were diagnosed in respectively $22 \%$ and $78 \%$. TMJ arthralgia (group III) was assigned unilaterally or bilaterally in respectively $28 \%$ and $51 \%$ of EDS population (Table 3). All subjects (100\%) were symptomatic for TMD (i.e. reporting pain or tenderness of masticatory muscles and TMJ, joint sounds and/or limitation or disturbance of mandibular movement), indicating a strong tendency for development of TMD in patients

Table 3 Individual temporomandibular disorders (TMD) diagnosis in Ehlers-Danlos syndromes (EDS) population $(n=31)$

\begin{tabular}{llll} 
& & \multicolumn{2}{l}{ Proportion (\%) } \\
\cline { 4 - 4 }$R C D / T M D^{\mathrm{a}}$ & Individual diagnosis $^{\mathrm{b}}$ & Unilateral & Bilateral \\
\hline Group I & $\begin{array}{c}\text { Myofascial pain } \\
\text { Group II }\end{array}$ & 83 & 78 \\
Gisk displacement & 22 & 51 \\
\hline $\begin{array}{c}\text { with reduction } \\
\text { Temporomandibular } \\
\text { joint arthralgia }\end{array}$ & 28 & \\
\hline
\end{tabular}

${ }^{\mathrm{a}} \mathrm{RCD} / \mathrm{TMD}$, research diagnostic criteria for temporomandibular disorders (18).

${ }^{\mathrm{b}} \mathrm{A}$ subject can be assigned at most one muscle diagnosis (Group I), whereas each joint may be assigned at most one diagnosis from Group II and one diagnosis from Group III (18). 
with EDS. Among these symptomatic subjects, only $22 \%$ were asking for treatment. Multiple TMD diagnoses were assigned in $89 \%$ of the individuals, of which $78 \%$ presented with three or more different RDC/TMD group diagnoses.

Oral parafunctions (grinding, clenching) were recorded in $28 \%$ of EDS population. There was no interrelation between occurrence of parafunctions and age, chronic pain report, presence of joint sounds and individual TMD diagnoses $(P=0.234)$. When analysing psychosocial history records, interpersonal factors (i.e. social or family problems; $P=0.013$ ) and emotional status (i.e. depression, anxiety; $P=0.046$ ) were assessed significantly more frequently in subjects with both myofascial pain and TMJ arthralgia diagnoses.

Recurrent TMJ dislocation was a constant finding in EDS population $(100 \%)$. Subjects with a high chronic pain score reported higher frequencies and longer duration of TMJ dislocations $(P<0.001)$, irrespective of age $(P=0.167)$ and EDS type $(P=0.284)$. There was no significant difference in factors provoking dislocations between subjects with high/low dislocation frequency $(P=0.458)$ or between different TMD diagnoses $(P=0.628)$.

\section{Oral mucosa}

Increased mucosal fragility was recorded in $74 \%$ of EDS population, without any significant difference between EDS types $(P=0.752)$. Local evidence of mucosal fragility (mostly bruises and regenerative patches) was present in $96 \%$ at two or more locations at the alveolar processes, in $78 \%$ at the hard palate, in $4 \%$ at the soft palate and in $35 \%$ at the cheeks. In $52 \%$, a combination of at least four locations was recorded, without any significant difference between locations at the lining or the masticatory mucosa $(P=0.238)$. The occurrence of bruising/grazing events was recorded in $61 \%$ as once or more a day, in $26 \%$ once or more a week, and in $13 \%$ less than once a week. The most prevalent initiating factors were mastication of food in $4 \%$, brushing teeth in $9 \%$, and a combination of both in $87 \%$. Subjects with increased mucosal fragility were found to have adopted special brushing techniques $(P=0.024)$, although brushing less frequently $(P=0.041)$. There was no difference in dental attendance between subjects with and without increased mucosal fragility in EDS $(P=0.465)$. In $52 \%$ of EDS population, regenerative patches appeared as pale red, circumscribed spots at locations with a high incidence of bruises and/or grazes, and hence might be interpreted as clinical manifestations of prolonged and deficient tissue healing (Fig. 2).

\section{Abnormalities of the dental hard tissues}

No abnormalities in tooth number were recorded in EDS population. Demarcated enamel opacities were found in $9.7 \%$ of EDS population. These defects exclusively presented in permanent premolars and could be related to caries experience in the deciduous dentition (local infectious aetiology). No abnormal tooth crown morphology was recorded. Root deformity was recorded in $6.5 \%$ of overall EDS population. The presence of

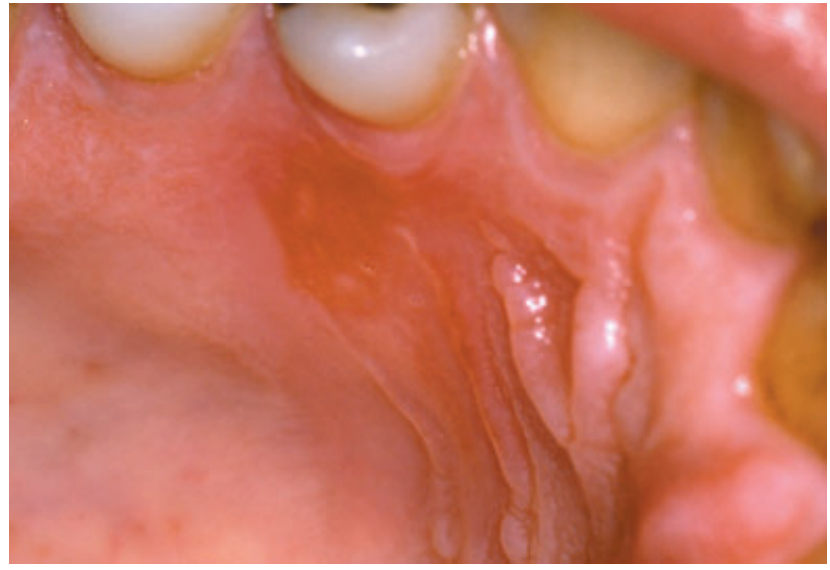

Figure 2 Regenerative patch at the frontal slope of the palate, indicative of recurrent grazing as a symptom of increased mucosal fragility, presenting in a patient with Ehlers-Danlos syndromes (EDS) hypermobility type (former EDS III).

abnormal pulp shape (13\% of EDS group) was found to be dependent on EDS type $(P<0.001)$, i.e. exclusively presenting in classical EDS. Demarcated calcification of the pulp (pulp stones) was recorded in 19\% of hypermobile EDS, in $78 \%$ of classical EDS, and was absent in vascular EDS (Fig. 3; Table 4). This was found to be significantly dependent on EDS type $(P=0.002)$.

\section{Caries experience}

The mean DMF-s score in EDS $(35.6 \pm 22.5)$ was significantly different from controls $(20.1 \pm 15.3)$ $(P=0.001)$, and was not related to EDS types $(P=0.085)$. There was no association between DMF-s score and dental attendance $(P=0.632)$, brushing habits $(P=0.424)$ and reports of recurrent mucosal erosions $(P=0.247)$ in EDS. DMF-s was significantly higher in EDS subjects with a high PII (rank correlation analysis; $r_{\mathrm{s}}=0.3 ; P=0.042$ ). This finding could be related to the presence of complaints of chronic limb pain (one-way ANOVA: $P<0.001$ ) and/or restraint joint mobility of the wrists $(P<0.001)$, resulting in poor oral hygiene. There was no statistically significant correlation between DMF-s and PII in controls $\left(r_{\mathrm{s}}=0.467 ; P=0.067\right)$. The mean dental care index $(F / D+F)$ was $0.81 \pm 0.24$ in EDS, and $0.92 \pm 0.11$ in controls, which yielded a statistically significant difference $(P=0.007)$.

\section{Oral cleanliness}

The mean PII in EDS was $2.3 \pm 0.6$, which was significantly different from controls (i.e. $1.7 \pm 0.6$; $P<0.001)$. No difference was found between EDS types $(P=0.1)$. There was no correlation between the individual PII and frequency of tooth brushing $(P=0.524)$, or reports of oral hygiene being influenced by the general condition $(P=0.160)$ in EDS population. Individuals with increased mucosal fragility brushed less frequently $(P=0.041)$, and had a significantly higher PII $(P=0.01)$. 

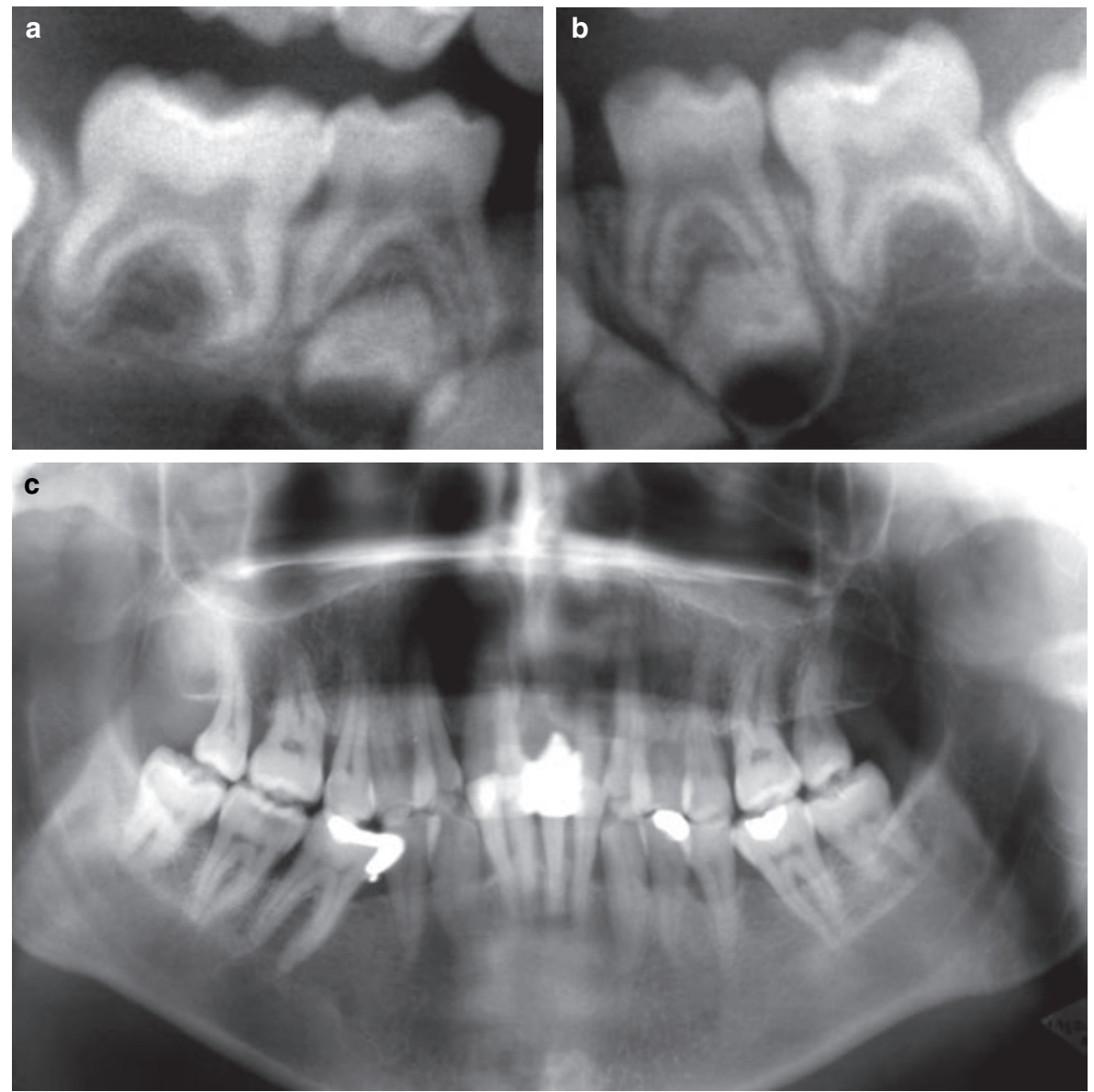

Figure 3 Root deformity (a, b), and abnormal pulp shape with progressive obliteration of the pulp chamber and pulp stones (c) are frequent findings in Ehlers-Danlos syndromes (EDS) classical type (former EDS I-II).

Table 4 Incidence of dentin defects in Ehlers-Danlos syndromes (EDS) population $(n=31)$

\begin{tabular}{llllc} 
& $\begin{array}{l}\text { Classical } \\
\text { EDS }(n=9)\end{array}$ & $\begin{array}{l}\text { Hypermobility } \\
\text { EDS }(n=16)\end{array}$ & $\begin{array}{l}\text { Vascular } \\
\text { EDS } \\
{[n(\%)}\end{array}$ & $\begin{array}{l}\text { Significance } \\
\text { P-value* }\end{array}$ \\
\hline $\begin{array}{l}\text { Root } \\
\text { deformity }\end{array}$ & $2(22)$ & 0 & 0 & 0.119 \\
$\begin{array}{c}\text { Abnormal } \\
\text { pulp shape }\end{array}$ & $9(100)$ & 0 & 0 & $<\mathbf{0 . 0 0 1}$ \\
$\begin{array}{c}\text { Pulp } \\
\text { calcification }\end{array}$ & $7(78)$ & $3(19)$ & 0 & $\mathbf{0 . 0 0 2}$ \\
\hline
\end{tabular}

*Considered significant at $P<0.05$ (chi-squared test).

\section{Periodontal status}

The mean pocket depth in EDS was $3.1 \pm 0.9$, which was significantly different from controls (i.e. $2.5 \pm 0.8$; $P=0.002)$. No difference could be established between EDS types $(P=0.093)$. The mean GI in EDS population was $1.2 \pm 0.4$. No significant difference was found between EDS and controls (i.e. $1.2 \pm 0.7$; $P=1.0)$ or among EDS types $(P=0.486)$. A CPITN $=$ II, indicating a high periodontal treatment need (improvement of oral hygiene and professional scaling needed), was present in $62 \%$ of EDS subjects. CPITN was significantly different among EDS types
$(P=0.047)$, i.e. a high CPITN was most frequent in hypermobile type, and less frequent in vascular EDS. Rank correlation analysis (Spearman's correlation coefficient) yielded significantly different interrelations in both groups between the presence of dental PII and GI inflammation $(P=0.847$ in EDS vs. $P<0.001$ in controls), gingival inflammation and mean pocket depth $(P=0.107$ in EDS vs. $P<0.001$ in controls $)$, and dental plaque and mean pocket depth $(P=0.424$ in EDS vs. $P=0.015)$. Regression analysis showed the mean pocket depth to be correlated with age in both EDS $(P=0.002)$ and controls $(P<0.001)$, but yielded a significantly different interrelation between gingival inflammation and age in both groups $(P=0.868$ in EDS vs. $P<0.001$ in controls).

\section{Discussion}

Ehlers-Danlos syndromes are inherited disorders of collagen biosynthesis that may present with specific manifestations in the oral cavity. In mild and undiagnosed cases, assessment of these manifestations may lead to EDS diagnosis. As metabolism of the most prevalent fibrillar collagens (type I, III and V) is altered in EDS (2), structural abnormalities of connective tissue may produce clinical symptoms in the different orofacial 
systems. In the TMJs, the fibrocartilage structure, supporting ligaments, the disc and retrodiscal tissues are composed mainly of collagen (types I, III and V) (30, $31)$. Hence, the effects of mutation in one of the genes encoding these proteins may be expressed as structural tissue alterations leading to joint laxity with internal joint derangement and dislocation (32). The present study indicates that hypermobility of the TMJs with recurrent TMJ dislocation is pathognomic for EDS, representing an important predisposing factor for TMD development. This finding is consistent with previous case reports $(14,33)$. The majority of subjects with EDS presents with a combination of myofascial pain, internal joint derangement and arthralgia of one or both the TMJs. Recently, a series of clinical TMJ hypermobility signs was proposed, based on an epidemiological study of a population partially consisting of subjects affected with EDS (34). As reduction of TMJ dislocation can cause intracapsular haemorrhage, recurrent dislocation may result in severe restraint of TMJ mobility (haemarthrosis). Treatment of TMD and TMJ dislocation (respectively haemathrosis) in EDS should be conservative and non-invasive, as high tissue fragility and extensive scar formation, being dominant features of the condition, may considerably interfere with restitution and healing processes $(12,35)$. In cases with recurrent TMJ dislocation - or patent TMJ hypermobility - and increased tissue fragility, the practitioner should be suspicious of a connective tissue involvement.

In contrast with previous reports (5-8), no abnormalities were recorded in tooth number and tooth crown morphology in classical and hypermobility EDS. During recent years an increasing number of genes have been identified that are involved in the regulation of tooth initiation and tooth morphogenesis. The majority of these genes are associated with the signalling pathways transmitting interactions between cells and epithelial and mesenchymal tissue layers. Mutations in several of these genes have been identified as causes of dental defects, mainly hypodontia and arrested tooth crown formation (36). As genetic and biochemical research did not yet elucidate a possible interrelation between mutation in collagen genes and early tooth development, the former dental defects most likely originate from those regulatory genes. Hence, previous reports on abnormal tooth number and/or abnormal tooth crown morphology in EDS might concern accidental findings rather than pathognomic signs related to collagen deficiency.

Increased mucosal fragility was a dominant finding in all examined EDS types. An interrelation between the location of the lesions and the type of the oral mucosa (lining or masticatory) could not be demonstrated, hence suggesting that neither the thickness, keratinization, or flexibility of the mucosal surface might influence the occurrence of tissue damage. As has previously been reported for skin fragility in $\operatorname{EDS}(1,2)$, structural alterations of the lamina propria, i.e. the connective tissue supporting the (oral) epithelium, might account for an increased fragility of the oral mucosa. The collagen in the lamina propria is primarily type I and type III, with type V occurring in inflamed or regener- ative tissue (37). Deficiency of collagen in the subepithelial connective tissue accounts for a decreased stretching and shears resistance of the epithelium, hence resulting in a high liability to 'tearing' of the mucosal tissues. Hence, extravasation of blood may occur in the lamina propria or between the lamina propria and oral epithelium, clinically presenting as a bruise, and the oral epithelium may be prone to easy grazing during mastication or brushing teeth. In addition, tissue regeneration may be hampered by a defective assembly of collagen fibrils. Recent findings also indicate that an abnormal oral mucosal reflectance may be a clinical marker of EDS (38). Abnormal pulp shape and moderate generalized pulp calcification (pulp stones) were frequent findings in (classical) EDS. Pulp calcification localized in one or a few teeth most commonly follows after traumatic injury, whereas generalized pulp obliteration is much more rare and can be related to age changes or to pulpal pathology, caused by long-standing chronic irritation, such as abrasion, erosion, periodontal disease, extensive dental restorations or carious lesions (39). Generalized pulp calcification is, moreover, present in dental anomalies such as dentinogenesis imperfecta or dentin dysplasia (40), or may be associated with systemic or genetic disease (41). The number and the size of the calcified bodies in classical EDS could not be proportionally related to the age of the individuals, or to the formerly mentioned long-standing irritating factors. Although the histogenesis of pulp calcifications is unknown, a relationship between pathological alterations in collagen molecules within the pulp and pulpal calcification has been suggested (39). Calcified spheroids may be found subcutaneously in about $30 \%$ of classical EDS, caused by calcification in hyalinized scar tissue (2, 41). Increased cross-linkage between (reparative) collagen molecules is thought to enhance the tendency for collagen fibres to calcify $(39,41)$, a process which may also account for the presence of pulpal calcification in EDS subjects, independent on age and/or tooth eruption stage.

The present study clearly demonstrates that oral health may be at risk in EDS. However, caries experience (DMF score) was significantly higher in EDS population compared with controls, treatment need was only $19 \%$ (dental care index $=0.81$ ). A high DMF-s score could individually be related to poor oral hygiene in EDS, which in return proved influenced by an increased mucosal fragility and chronic limb pain in the presence of restraint joint mobility (mostly wrists, interfering with proper brushing habits). The latter features appeared to hamper proper oral hygiene techniques. Loss of gingival attachment was significantly greater in EDS population, and was found to be unrelated to gingival bleeding tendency and oral hygiene. These findings were contradictory to controls, and hence might result from specific structural (decreased resistance to mechanical and bacterial assaults) and/or biochemical (deficient healing or restitutional capacities) alterations of collagens in EDS, leading to rapid periodontal breakdown in the presence of pathogenic bacteria. In previous studies, types I, III and V collagen have been identified in gingival 
connective tissue and the periodontal ligament. Type I collagen is the principal extracellular matrix protein in periodontal soft connective tissues (42), and is found in association with type III collagen throughout the tissue. Type III collagen is a major constituent of vascular walls (43), whereas type V collagen has a more filamentous distribution, and has been observed in association with type I collagen fibril formation (44). Type I collagen also accounts for $95 \%$ of total cementum collagens, especially presenting in Sharpey's fibres, and was found to be coated by type III collagen (45). In periodontitis, the collagens become more soluble, and the ratios of collagen types are altered. Furthermore, the amount of type $\mathrm{V}$ collagen increases and a new, unstable collagen, type I trimer, may appear (46). There does not appear to be a change in localization and distribution of constituent collagen types in periodontitis (47). Restitution of the periodontal tissues after root scaling may be seriously compromised in EDS by deficient processing of new collagen proteins and fibrils, leading to a decreased regenerative capacity of connective tissues. The major challenge in contemporary periodontal therapy, i.e. re-establishing soft tissue attachment to newly formed cementum on the root surface (48), may be hampered by defective interdigitation of newly assembled (abnormal) collagen fibres to old fibres on the root surface (49). This may lead to failure of restitution of the periodontal ligament in disorders with deficient collagen biosynthesis.

The present study demonstrates a clear interrelation between deficiency of specific collagen types and clinical manifestations in the different orofacial structures (Table 5). Previous reports on oral manifestations of collagen I deficiency in subjects affected with osteo-

Table 5 Expression of deficiency of collagen I, III and V in orofacial structures [Ehlers-Danlos syndromes (EDS)]

\begin{tabular}{|c|c|c|}
\hline Structure & Collagen types & $\begin{array}{l}\text { Clinical expression of } \\
\text { collagen deficiency }\end{array}$ \\
\hline Bone & I-III-V & $\begin{array}{l}\text { Slow socket healing after } \\
\text { tooth extraction } \\
\text { Deficient restitution after } \\
\text { periodontal treatment }\end{array}$ \\
\hline $\begin{array}{l}\text { Temporomandibular } \\
\text { joint (TMJ) }\end{array}$ & I-III-V & $\begin{array}{l}\text { TMJ hypermobility with } \\
\text { recurrent dislocations } \\
\text { High susceptibility to } \\
\text { temporomandibular } \\
\text { disorders development } \\
\text { High susceptibility to } \\
\text { TMJ haemathrosis with } \\
\text { consequent restraint } \\
\text { TMJ mobility }\end{array}$ \\
\hline Tooth/dentin & I & Root deformity \\
\hline Tooth/pulp & I-III & $\begin{array}{l}\text { Abnormal pulp chamber } \\
\text { Pulp calcification }\end{array}$ \\
\hline Mucosa & I-III-V & $\begin{array}{l}\text { Increased fragility with } \\
\text { prolonged healing } \\
\text { Increased bleeding tendency } \\
\text { Tearing of sutures }\end{array}$ \\
\hline $\begin{array}{l}\text { Periodontal } \\
\text { ligament }\end{array}$ & I-III-V & $\begin{array}{l}\text { Rapid loss of attachment } \\
\text { (also juvenile variants) } \\
\text { Deficient restitution after } \\
\text { professional root scaling }\end{array}$ \\
\hline
\end{tabular}

genesis imperfecta (OI), mainly documented on dentin defects (dentinogenesis imperfecta) in the deciduous and permanent dentition (50-56). A high incidence of class III dental malocclusion, anterior and posterior crossbites, and ectopic tooth eruption were also reported in OI, but these characteristics most likely seem related to abnormal growth and development of the craniofacial bones as a result of abnormal collagen I biosynthesis (54). In general, the clinical and molecular characteristics of collagen I disorders (e.g. OI and dermatosparaxis EDS type) are strictly determined by the causal gene mutation, which interferes in specific pathways in collagen synthesis (57). These distinct interferences may result in a wide range of clinical features in the connective tissues (58). The present findings are consistent with the conclusion that orofacial manifestations in collagen deficiency are entirely dependent on the affected collagen type and on the molecular/genetic basis of the disease.

When a practitioner considers dental treatment for a patient with EDS, a number of tissue responses and precautions should be anticipated. Oral mucosae may be fragile and easily bruised. Gingival tissues may be more liable to injury and periodontal disease, prompting oral pathologists to look for (juvenile) cases of rapidly progressive periodontal breakdown. The bruising and bleeding tendencies have been attributed to increased fragility of vessel walls $(1,2)$. Because of inborn problems with tissue repair, slow and deficient healing after tooth extraction and periodontal treatment, slowly forming new bone in sockets, and soft tissue scarring may occur $(6,7,17)$. The dental practitioner may have to be careful to prevent dislocation of the TMJs during treatment $(9,12)$. Preventive treatment has to address proper oral hygiene methods in relation to the subject's restraint joint mobility and/or mucosal bruising tendency. Pulp chamber deformity and progressive pulp obliteration may compromise endodontic treatment.

\section{References}

1. Beighton P, De Paepe A, Danks D et al. International nosology of heritable disorders of connective tissue, Berlin, 1986. Am J Med Genet 1988; 29: 581-94.

2. Beighton P, De Paepe A, Steinmann B, Tsipouras P, Wenstrup RJ. Ehlers-Danlos syndromes: revised nosology, Villefranche, 1997. Am J Med Genet 1998; 77: 31-7.

3. OMIM Entry (Online Mendelian Inheritance in Man). Available at: http://www3.ncbi.nlm.nih.goventrez/query. fcgi? $\mathrm{db}=\mathrm{OMIM}$. (Accessed in November 2004)

4. Gorlin RJ, Cohen MM, Jr, Levin LS. Syndromes of the Head and Neck, 3rd edn. New York, NY, USA: Oxford University Press, 1990; 267-73.

5. Barabas GM, Barabas AP. The Ehlers-Danlos syndrome: a report of the oral and haematological findings in nine cases. Brit Dent J 1967; 123: 473-9.

6. Fridrich KL, Fridrich HL, Kempf KK, Moline DO. Dental implications in Ehlers-Danlos syndrome. Oral Surg Oral Med Oral Pathol 1990; 69: 431-5.

7. Letourneau Y, Perusse R, Buithieu H. Oral manifestations in Ehlers-Danlos syndrome. J Can Dent Assoc 2001; 67: $330-4$. 
8. Melamed Y, Barkai G, Frydman M. Multiple supernumerary teeth and Ehlers-Danlos syndrome: a case report. J Oral Pathol Med 1994; 23: 88-91.

9. Goodman RM, Allison ML. Chronic temporomandibular joint subluxation in Ehlers-Danlos syndrome: report of case. J Oral Surg 1969; 27: 659-61.

10. Sacks H, Zelig D, Schabes G. Recurrent temporomandibular joint subluxation and facial ecchymosis leading to diagnosis of Ehlers-Danlos syndrome. J Oral Maxillofac Surg 1990; 48: 641-7.

11. Westling L. Temporomandibular joint dysfunction and systemic joint laxity. Swed Dent J Suppl 1992; 81: 71-9.

12. McDonald A, Pogrel AP. Ehlers-Danlos syndrome: an approach to surgical management of temporomandibular joint dysfunction in two cases. J Oral Maxillofac Surg 1996; 54: 761-5.

13. Miller VJ, Zeltser R, Yoeli Z, Bodner L. Ehlers-Danlos syndrome, fibromyalgia and temporomandibular disorder: report of an unusual combination. J Craniomandib Pract 1997; 15: 267-9.

14. Norton LA, Assael LA. Orthodontic and temporomandibular joint considerations in treatment of patients with Ehlers-Danlos syndrome. Am J Orthod Dentofac Orthop 1997; 111: 75-84.

15. Hagberg C, Berglund B, Korpe L, Anderson-Norinder J. Ehlers-Danlos syndrome focusing on oral problems: a questionnaire study. Orthod Craniofac Res 2004; 7: 17885.

16. Reichert S, Riemann D, Plashka B, Maculla HK. Earlyonset periodontitis in a patient with Ehlers-Danlos syndrome type VIII. Quintessence Int 1999; 30: 785-90.

17. Perez LA, Al-Shammari KF, Giannobile WV, Wang HL. Treatment of periodontal disease in a patient with EhlersDanlos syndrome: a case report and literature review. J Periodontol 2002; 73: 564-70.

18. Pope FM, Komorowska A, Lee KW et al. Ehlers-Danlos syndrome type I with novel dental features. J Oral Pathol Med 1992; 21: 418-21.

19. Karrer S, Landthaler M, Schmalz G. Ehlers-Danlos type VIII. Review of the literature. Clin Oral Investig 2000; 4: 66-9.

20. WHO. Oral Health Surveys: Basic Methods, 4th edn. Geneva, Switzerland: World Health Organization, 1997.

21. LeResche L. Axis I: Clinical TMD conditions. In: Dworkin SF, LeResche L, eds. Research Diagnostic Criteria for Temporomandibular Disorders: Review, Criteria, Examinations and Specifications, Critique. J Craniomand Dis Fac Oral Pain 1992; 6: 327-30.

22. Widmer GW. Examination and history data collection. In: Dworkin SF, LeResche L, eds. Research Diagnostic Criteria for Temporomandibular Disorders: Review, Criteria, Examinations and Specifications, Critique. J Craniomandib Disord 1992; 6: 335-45.

23. Okeson JP. Orofacial Pain: Guidelines for Assessment, Diagnosis, and Management - The American Academy of Orofacial Pain. Chicago, IL, USA: Quintessence, 1996; 134.

24. Mitropoulos C, Pitts N. BASCD Trainers' Pack for Caries Prevalence Studies 1992/1993. University of Dundee: Dental Health Services Research Unit, 1993.

25. Jackson D. Measuring restorative dental care in communities. Br Dent $J$ 1973; 134: 385-8.

26. Lang NP. Commonly used indices to assess oral hygiene and gingival and periodontal health and diseases. In: Proceedings of the European Workshop on Mechanical Plaque Control, May 9-12, 1998. Chicago, IL, USA: Quintessence, 1998; 52-69.
27. Ainamo J, Barmes D, Beagrie G, Cutress T, Martin J Sardo-Infirri J. Development of the WHO community periodontal index for treatment need (CPITN). Int Dent $J$ 1982; 32: 281-91.

28. Clayton D, Hills M. Statistical Models in Epidemiology. Oxford, UK: Oxford University Press, 1993.

29. Petrie A, Sabin C. Medical Statistics at a Glance. Oxford, UK: Blackwell Science Ltd, 2000.

30. Gage JP, Virdi AS, Triffit JT, Howlett CR, Francis MJO. Presence of type III collagen in disk attachments of human temporomandibular joints. Arch Oral Biol 1990; 35: 283-8.

31. Visnapuu V, Peltomäki T, Säämänen A-M, Rönning O. Collagen I and II mRNA distribution in the rat temporomandibular joint region during growth. J Craniofac Genet Dev Biol 2000; 20: 144-9.

32. Graham R. Joint hypermobility and genetic collagen disorders: are they related? Arch Dis Child 1999; 80: 18891.

33. Vitagliano J, Norton LA, Assael LA. Mandibular joint orthodontic and dental findings in EDS: report of a survey. Loose Connections 1995; 10: 1-4.

34. De Coster PJ, Van den Berghe L, Martens C. Generalized joint hypermobility and temporomandibular disorders: inherited connective tissue disease as a model with maximum expression. J Orofac Pain, in press.

35. Weinberg $\mathbf{J}$, Doering $\mathrm{C}, \mathrm{McF}$ arland EG. Joint surgery in Ehlers-Danlos patients: results of a survey. Am J Orthop 1999; 28: 406-9.

36. Thesleff I. Genetic basis of tooth development and dental defects. Acta Odontol Scand 2000; 58: 191-4.

37. Squier CA, Finkelstein MW. Oral mucosa. In: Nanci A, ed. Ten Cate's Oral Histology, 6th edn. St Louis, MO, USA: CV Mosby, 2003; 329-75.

38. Parrini S, Bellosi A, Barducci A, Biancardi G, Latini G, De Felice C. Abnormal oral mucosal reflectance: a new clinical sign of Ehlers-Danlos syndrome. Oral Surg Oral Med Oral Pathol Oral Radiol Endod 2004; 97: 335-8.

39. Cohen S, Burns RC. Pathways of the Pulp, 5th edn. St Louis, MO, USA: CV Mosby, 1998; 341-5.

40. Witkop CJ, Jr. Amelogenesis imperfecta, dentinogenesis imperfecta and dentin dysplasia revisited: problems with classification. J Oral Pathol 1988; 17: 547-53.

41. Beighton P, Thomas ML. The radiology of the EhlersDanlos syndrome. Clin Radiol 1969; 20: 354-61.

42. Narayanan AS, Clagett JA, Page RC. Effect of inflammation on the distribution of collagen types I, III, and V and type I trimer and fibronectin in human gingivae. J Dent Res 1985; 64: 1111-6.

43. Romanos G, Schroter-Kermani C, Hinz N, Bernimoulin JP. Immunohistochemical distribution of the collagen types IV, V, VI and glycoprotein laminin in the healthy rat, marmoset and human gingivae. Matrix 1991; 111: 125-32.

44. Narayanan AS, Page RC. Synthesis of type V collagen by fibroblasts derived from normal, inflamed and hyperplastic human connective tissues. Collagen Rel Res 1983; 5: 297-304.

45. Bartold PM, Laurence JW, Narayanan AS. Molecular and cell biology of the gingiva. Periodontol 2000 2000; 24: 28 55.

46. Ruoslashti E, Yamaguchi Y. Proteoglycans as modulators of growth factor activities. Cell 1991; 64: 867-9.

47. Salonen J, Domenicucci C, Goldberg HA, Sodek J. Immunohistochemical localization of SPARC (osetonectin) and denaturated collagen and their relationship to remodelling in rat dental tissues. Arch Oral Biol 1990; 35: $337-42$. 
48. Karring T, Lindhe J, Cortellini P. Regenerative periodontal therapy. In: Lindhe J, ed. Clinical Periodontology and Implant Dentistry, 3rd edn. Copenhagen: Munksgaard, 1998; 597-646.

49. Barthold PM. Turnover in periodontal connective tissues: dynamic homeostasis of cells, collagen and ground substances. Oral Dis 1995; 1: 238-53.

50. Schwartz S, Tsipouras P. Oral findings in osteogenesis imperfecta. Oral Surg Oral Med Oral Pathol 1984; 57: $161-7$.

51. Lukinmaa PL, Ranta H, Ranta K, Kaitila I. Dental findings in osteogenesis imperfecta: I. Occurrence and expression of type I dentinogenesis imperfecta. J Craniofac Genet Dev Biol 1987; 7: 115-25.

52. Levin LS. Osteogenesis imperfecta type I with unusual dental abnormalities. Am J Med Genet 1988; 31: 92132.

53. Aldred MJ. Unusual dentinal changes in dentinogenesis imperfecta associated with osteogenesis imperfecta: a case report. Oral Surg Oral Med Oral Pathol 1992; 73: $461-4$.

54. O'Connell AC, Marini JC. Evaluation of oral problems in an osteogenesis imperfecta population. Oral Surg Oral Med Oral Pathol Oral Radiol Endod 1999; 87: 189-96.

55. Stephen LXG, Beighton P. Dental management of severe dentinogenesis imperfecta in a mild form of osteogenesis imperfecta. J Clin Pediatr Dent 2002; 26: 131-6.
56. Malmgren B, Norgren S. Dental aberrations in children and adolescents with osteogenesis imperfecta. Acta Odontol Scand 2002; 60: 65-71.

57. De Coster PJ, Malfait F, Martens L, De Paepe A. Unusual oral findings in dermatosparaxis (Ehlers-Danlos syndrome type VIIc). J Oral Pathol Med 2003; 32: 568-70.

58. Malfait F, De Coster PJ, Hausser I et al. Follow-up history and unusual oro-dental findings in three patients with human dermatosparaxis (Ehlers-Danlos syndrome type VIIC). Am J Med Genet, in press.

\section{Acknowledgements}

The authors wish to thank Hilde De Vree, Department of Periodontology, Ghent University, for training and calibration of periodontal indices, Prof. (Dr) Linda Van den Berghe, Unit for Temporomandibular Disorders and Orofacial Pain, Ghent University, for training and calibrating TMD examination methods, and Prof. (Dr) Georges Van Maele, Department of Medical Informatics, Ghent University, for monitoring the statistical models and processing the research data. Peter J. De Coster is a Research Fellow, affiliated to the Centre for Special Care and PaeCaMed Research, Dental School, Ghent University Hospital, Belgium. The present study is part of his doctoral research project entitled: 'Expression of Collagen and Fibrillin Deficiency in Orofacial Structures: An Epidemiological, Morphometrical and Ultrastructural Analysis'. 